\title{
Telemedizin bringt Krankenhäusern neue Möglichkeiten Zusammenarbeit zwischen den Sektoren wird immer wichtiger
}

\begin{abstract}
Prof. Dr. Mark Dominik Alscher, Facharzt für Innere Medizin - Nephrologie, ist Ärztlicher Direktor am Robert-Bosch-Krankenhaus in Stuttgart. Zugleich führt er gemeinsam mit seinem Kollegen Prof. Dr. Michael Bamberg, Vorstandsvorsitzender des Universitätsklinikums Tübingen, den Vorsitz der AG Telematik des Gesundheitsforums Baden-Württemberg. Die Arbeitsgruppe wurde 2011 eingerichtet, um die sektorenund professionsübergreifende regionale Vernetzung von Versorgungsangeboten durch Telemedizin zu unterstützen. Im Vordergrund steht der Transfer von Erkenntnissen der medizinnahen IT-Forschung in die Versorgungsrealität. Welchen Nutzen können Krankenhäuser hieraus ziehen?
\end{abstract}

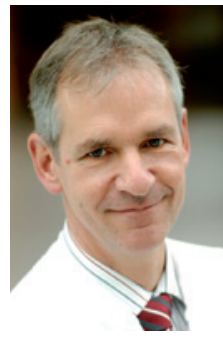

? Seit wann befassen Sie sich mit Telemedizin? Was begründet Ihr Engagement? Prof. Mark Dominik Alscher: Ohne Informationstechnologie (IT) könnte ich meinen Arbeitsalltag nicht bewältigen. Speziell für das Thema Telematik kam der erste große Impuls für das Krankenhaus durch das Projekt „Partnership for the Heart“, an dem wir uns 2007 gemeinsam mit der Charité in Berlin beteiligt haben. Das Projekt wurde damals vom Bundeswirtschaftsministerium gefördert. Industriepartner und Krankenkassen waren auch dabei. Über telemedizinische Instrumente sollte die Betreuung von Patienten mit Herzschwäche verbessert werden, d.h. die Patienten in ihrer häuslichen Umgebung zu versorgen.

Welche telemedizinischen Trends sehen Sie in Baden-Württemberg? Prof. Alscher: Baden-Württemberg ist offen für Innovationen. Das Besondere ist hier, dass die Förderung der versorgungsrelevanten Projekte über das Wissenschaftsministerium erfolgt. Das Sozialministerium ist eingebunden, aber nicht federführend. Man könnte fast von einem baden-württembergischen Modell sprechen, bei dem Qualitätsaspekte der Forschung und Versorgung in den Vordergrund rücken. Ein Stichwort ist die evidenzbasierte Medizin. Des Weiteren sehen wir den Bürger im Fokus unseres Bemühens. Wir wollen Telemedizin aus dessen Sicht entwickeln, sodass sie ihm den größtmöglichen Nutzen bringt.

Welche Entwicklungen sind für Krankenhäuser relevant?
Prof. Alscher: Für Krankenhäuser wird die Zusammenarbeit zwischen den Sektoren immer wichtiger. Mittlerweile werden viele Leistungen ambulant erbracht, die wir früher ausschließlich stationär erbringen konnten. Um eine hohe Versorgungsqualität aufrecht zu halten, ist es daher notwendig, dass wir als hoch spezialisierte Zentren unsere Expertise sektorenübergreifend zur Verfügung stellen und eine qualitativ gute Facharztausbildung ermöglichen. Telemedizin hilft, die räumlichen Distanzen zu überwinden, und fördert dadurch den Wissenstransfer und die Zusammenarbeit. Die Krankenhäuser müssen lernen, die Versorgungskette vom ambulanten in den stationären Sektor und wieder zurück aus Sicht des Patienten zu sehen, und hierbei ihre neue Rolle finden und annehmen.

Über welche Telematik-Infrastruktur verfügt Ihr Krankenhaus?

Prof. Alscher: In Kürze werden wir unser Dokumentationssystem komplett auf eine elektronische Patientenakte umgestellt haben, sodass die Arbeit auf den Stationen real papierlos erfolgt. Die Umsetzung erfordert ein gutes Projektmanagement, da Krankenhäuser eher strukturkonservativ sind und sehr sensibel auf Patientenbelange reagieren. Die neue Technologie darf nicht zulasten der Patientensicherheit gehen. Doch selbst mit der elektronischen Akte ist der Prozess der Digitalisierung nicht abgeschlossen. Wir müssen am Ball bleiben. Die Einbindung elektronischer Tools in die Patientenversorgung ist ein permanenter Prozess, vor allem wenn es um die sektoren- und einrichtungsübergreifende Zusammenarbeit geht. Wir gehören zum Beispiel einem RadiologieNetzwerk an und holen darüber externe
Expertise ein bzw. stellen unsere Expertise zur Verfügung. Wir teilen so hochspezialisiertes Wissen, das nicht flächendeckend zur Verfügung steht. Außerdem haben wir intern ein Doc-to-Doc-System eingerichtet, um mittels Videokonferenz die Kompetenzen unserer drei Standorte zeitnah nutzen zu können. Für Patienten mit COPD hat unser Haus ein Telemedizinisches Zentrum aufgebaut, welches die Versorgung besonders schwer erkrankter Patienten bundesweit unterstützt.

Hat sich mit der neuen Technologie die Arbeitsorganisation in Ihrem Haus verändert? Wie haben Ärzte und Pflegekräfte hierauf reagiert?

Prof. Alscher: Am Anfang gab es gelegentlich Bedenken, ob unsere Belegungszahlen durch die sektorenübergreifende Ausrichtung rückläufig sein würden. Dies hat sich mittlerweile gelegt. Die Vorteile einer besseren Betreuung der Patienten durch eine schnelle und eindeutige Datenübermittlung überwiegen. Die neuen Technologien bei Videokonferenzen und im RadiologieNetzwerk ließen sich recht schnell in die Arbeitsorganisation integrieren. Die meisten Tools waren selbsterklärend, zusätzlich gab es Anwenderschulungen. Natürlich haben wir auch die Mitarbeiter des Telemedizinischen Zentrums auf ihre Aufgabe vorbereitet. Sie bilden innerhalb des Hauses noch eine Art „Insel“, da sie mit ihrem Knowhow die ambulante Behandlung aus der Ferne unterstützen.

\section{Welche telemedizinischen Ziele} verfolgen Sie für Ihr Haus? Was planen Sie im nächsten Jahr zu realisieren? Prof. Alscher: Wir beteiligen uns an Ausschreibungen des Landes, um weitere telemedizinische Innovationen einzuführen, zum Beispiel für pflegebedürftige Patienten in häuslicher Umgebung oder für geriatrische Patienten. Darüber hinaus entwickeln wir Vorschläge für Selektivverträge, die wir den Krankenkassen anbieten. Auch unsere telemedizinische Betreuung von COPD-Patienten wird über einen Integrierten Versorgungsvertrag finanziert.

Welche Hürden gilt es zu überwinden? 
Prof. Alscher: Gegenwärtig lässt sich Telemedizin nur über externe Fördermittel realisieren. Sie kommt daher immer nur einer kleinen Patientengruppe zugute. Für die Regelversorgung ist es notwendig, dass es eine Gebührenziffer zur Abrechnung für den ambulanten und den stationären Sektor gibt. Eine OPS-Abrechnungsziffer für telemedizinische Leistungen gibt es bereits für die neurologische Komplexbehandlung eines Schlaganfallpatienten.

\section{? Wo sehen Sie den größten Nutzen, den Krankenhäuser aus der Telematik- AG ziehen können?}

Prof. Alscher: Die Telematik-AG hilft Anfangswiderstände gegenüber Technikinnovationen $\mathrm{zu}$ überwinden und leistet eine Anschubfinanzierung für neue Projekte. Dies ist wichtig, um exemplarisch aufzuzeigen, welche neuen Möglichkeiten es für den Erhalt einer qualitativ hochwertigen Versorgung auch in Regionen mit geringer Arztdichte gibt und welche neuen Aufgaben das Krankenhaus hierbei übernehmen kann. Wir bereiten dadurch den Weg vor, damit es den Krankenkassen leichter fällt, in die Finanzierung von Telemedizin einzusteigen. Krankenhäuser können zwar interne Pro- zessverbesserungen aus dadurch eingesparten Mitteln bezahlen, nicht jedoch die Kosten für eine sektorenübergreifende Telematik-Infrastruktur, die die Kompetenzen der verschiedene Professionen und Einrichtungen zusammenführt und Patienten im Selbstmanagement ihrer Krankheit unterstützt.

Herr Prof. Alscher, herzlichen Dank für das Gespräch!

Das Interview führte Dr. Adelheid Weßling, Düsseldorf. 